\title{
HM4All: A Vital Signs Monitoring System based in Spatially Distributed ZigBee Networks
}

\author{
Helena Fernández-López, José A. Afonso and J. H. \\ Correia \\ Industrial Electronics Engineering Department \\ University of Minho, Guimarães, Portugal \\ \{hlopez, jose.afonso, higino.correia\}@dei.uminho.pt
}

\author{
Ricardo Simões \\ Institute of Polymers and Composites \\ University of Minho, Guimarães, Portugal, and \\ Cávado and Ave Polytechnic Institute, \\ Campus do IPCA, 4750-820 Barcelos, Portugal \\ rsimoes@dep.uminho.pt; rsimoes@ipca.pt
}

\begin{abstract}
Low power consumption and small footprint make ZigBee based devices well suited for personal healthcare applications, being a promising alternative to general care patient monitoring. However, their use in a healthcare facility to monitor several mobile patients poses several difficulties, mainly because this protocol was primarily designed to operate in low data rate scenarios. This paper introduces HM4All, a remote vital signs monitoring system, and presents a prototype system being deployed in a hospital internment floor. Its architecture, original network topology, software applications and wireless sensors are described.
\end{abstract}

Keywords-HM4All; Vital signals monitoring; ZigBee; wireless sensor networks.

\section{INTRODUCTION}

According to the Sixth Annual HealthGrades Patient Safety in American Hospitals Study, failure to rescuet events are among the medical errors of highest incidence rates in American hospitals, accounting for a total of 14,903 deaths between 2005 and 2007 [1]. Ideally, all hospital patients should have one or more vital signs monitored to reduce care response delay in case an adverse event occurs [2]. Still, this scenario is contrary to what presently occurs in most hospitals, where typically few patients out of the intensive and intermediate care units have their vital signs monitored. To extend the monitoring services to most hospital patients, monitoring systems must be pervasive, non-intrusive and use low cost consumables.

Continuous health monitoring based on wireless sensor networks (WSNs) can greatly benefit general care patients, including mobile in-patients. WSNs consist of spatially distributed devices containing sensor units used to acquire and process data and communicate with each other using a radiofrequency channel. These networks are characterized by several features, such as self-organizing capabilities, shortrange broadcast communication and multi-hop routing, frequently changing topology due to fading and node failures, and power limitations [3].

To be feasible, each sensor network must consist of unobtrusive sensor nodes that consume very little power, since

tAccording to the American Agency for Healthcare Research and Quality (http://www.psnet.ahrq.gov/glossary.aspx), "failure to rescue" refers to the inability of conducting actions that could have prevented a clinically important deterioration, such as death or permanent disability, from a complication of an underlying illness or a complication of medical care that developed on their watch. It may reflect the quality of monitoring, the effectiveness of actions taken once early complications are recognized, or both.

PERVASIVEHEALTH 2010, March 22-25, Munchen, Germany

Copyright (C) 2010 ICST 978-963-9799-89-9

DOI 10.4108/ICST.PERVASIVEHEALTH2010.8836 they must be powered by batteries. On the other hand, the reliance on these systems depends on the satisfaction of quality of service (QoS) requirements, such as sustainable throughput, small delay and high reliability. The main difficulty arises from the fact that some sensors must be sampled quite often, generating a large amount of data and, consequently, requiring the network to operate under high load, which is not common in typical WSN scenarios. Therefore, a careful network design is required, as will be further discussed, to assure that QoS requirements are achieved.

The usage of standard-based communication technologies with healthcare oriented profiles can offer several benefits, such as:

- Standard protocol stacks implementations are reliable and can considerably reduce development costs.

- Standard-based radios and integrated communication modules are cheaper than customized solutions.

- Medical sensors from a variety of manufacturers can coexist and exchange information.

In this paper, we introduce HM4All (Health Monitor for All), a prototype vital signs monitoring system that will be deployed in an internment floor of a private hospital in Portugal. HM4All is based on WSN technologies and standardbased protocols, and was designed to allow remote monitoring of ECG (electrocardiography), $\mathrm{SpO} 2$ (oxygen saturation in the blood), and skin temperature. Out-patients recovering at home can also be monitored by specialized healthcare providers in the hospital. Additionally, we have developed the infrastructure necessary to monitor patients anywhere using a personal digital assistant (PDA).

The paper is organized as follows. In the next section, we present a short discussion about the IEEE 802.15.4 and ZigBee protocols. In Section III, we introduce the overall system architecture and the network topology adopted. Section IV presents the prototype system being deployed, including the specific topology, and the wireless sensors and software applications developed for this work. In Section V, we discuss some results already achieved. In the next section, we present the related work and, finally, in the last section, the conclusions are presented. 


\section{IEEE 802.15.4 AND ZigBEE PROTOCOLS}

HM4All WSN is based on the 802.15.4 [4] and ZigBee [5] protocols, which provide the network infrastructure required for WSN applications. The 802.15.4 standard was developed by the IEEE, and defines the physical (PHY) and medium access (MAC) layers. The PHY layer is responsible for data transmission and reception and defines three possible frequency bands. The higher frequency band ranges from 2400 $\mathrm{MHz}$ to $2483.5 \mathrm{MHz}$, divided into sixteen $2 \mathrm{MHz}$ channels that operate at a rate of $250 \mathrm{kbps}$. The MAC layer handles the access to the physical radio channel using a contention-based CSMA-CA algorithm.

The ZigBee protocol, developed by the ZigBee Alliance, stands on top of the 802.15.4 and defines the network and application layers. Both protocols are intended for applications that require long battery life, low cost, small footprint, and mesh networking to support communication between large numbers of devices in an interoperable and multi-application environment. The network layer is responsible for routing frames to their intended destinations and provides functionalities such as network starting and newly associated devices address assignment and mechanisms to join and leave the network. It also provides an interface to the application layer, which holds application objects and provides mechanisms for discovering and binding devices [5].

The stack architecture is shown in Figure 1, where the Application Profile is shown on top of ZigBee and 802.15.4 stacks. Applications must conform to public or manufacturer specific profiles. Public profiles are defined by the ZigBee Alliance, and provide a description of the devices supported for a specific application together with the messaging scheme. They guarantee end-to-end interoperability between products running the same public application profile [6].

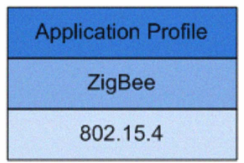

Figure 1. ZigBee protocol stack

\section{SYSTEM ARCHITECTURE AND TOPOLOGY}

\section{A. Architecture}

The overall system architecture is shown in Figure 2 ZigBee-based wearable sensors used by in-patients associate to one of the WSNs in operation in the hospital floor to continuously send vital sign data to one of the network coordinators (only one WSN is shown, but several can coexist). Data is sent through a serial interface to the ZigBee-to-Wi-Fi Gateway, where they are processed and sent, through the Wi-Fi infrastructure to the Data Server. Patients' vital signs can be presented by any Web browser running in any computer (monitoring station). More than one monitoring station can be used to visualize data.

Out-patients, recovering at home, can have their vital signs monitored as well, provided they have the ZigBee infrastructure and the required applications running on a personal computer with access to the Internet. Healthcare

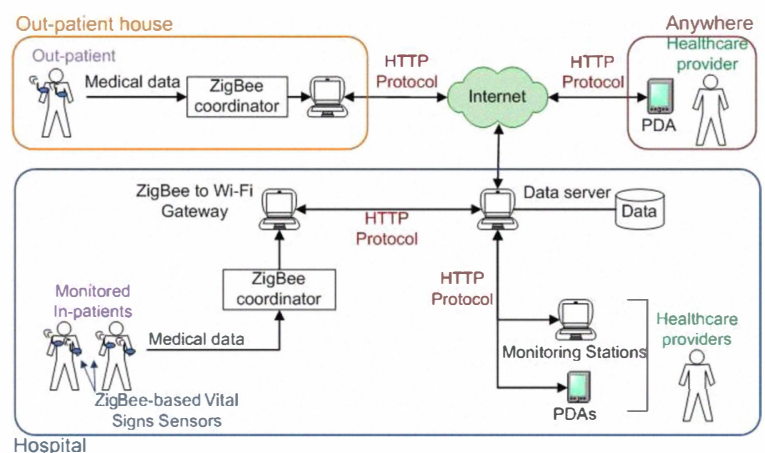

Figure 2. Overall system architecture.

providers can observe patients' vital signs and, in the future, they will be able to receive alerts in a PDA.

\section{B. Network Topology}

Two possible network topologies were considered: one based on body area networks (BANs) and the other consisting of spatially distributed networks, an original concept we have decided to explore. In the first case, a portable device worn by the monitored patient (for instance, a PDA with ZigBee and Wi-Fi communication capabilities) works as the network coordinator and as the gateway. In the second case, sensors associate with one of the network coordinators or routers to be able to send data. No additional patient device is needed.

If a BAN had been used, every monitored patient would carry a portable device, what would be costly and more intrusive. Additionally, every device should have batteries recharged or substituted quite often. On the other hand, considering that Wi-Fi networks are quite pervasive in hospital environments, this solution has the potential of extending the system connectivity to any area with Wi-Fi access.

To make the system less intrusive to patients, we have chosen to use spatially distributed networks, each one operating in distinct channels (ZigBee can operate in 16 different channels). Besides of the advantages already mentioned, this topology is highly scalable, flexible and robust. If propagation conditions worsen (if, for instance, walls or ceilings are coated with metallic sheets) or if the network gets more dense (for instance, if, instead of a single patient, two patients are accommodated in each room), more networks can be deployed to assure adequate quality of service.

\section{PROTOTYPE SYSTEM}

\section{A. Topology}

The prototype system must be able to monitor up to six patients. Considering this requisite, the specific topology shown in Figure 3, with two WSNs operating in two different channels, will be used. Sensors will be able to associate with any coordinator $(\mathrm{C} 1$ or $\mathrm{C} 2)$ or router, the one that offers the better signal quality. Blue and red arrows indicate possible communication paths between network elements. Both coordinators are connected to one ZigBee-to-Wi-Fi gateway which will send data to a Web-based application (the green arrows indicate Wi-Fi connections) that, in this particular deployment, will run in the monitoring station. 


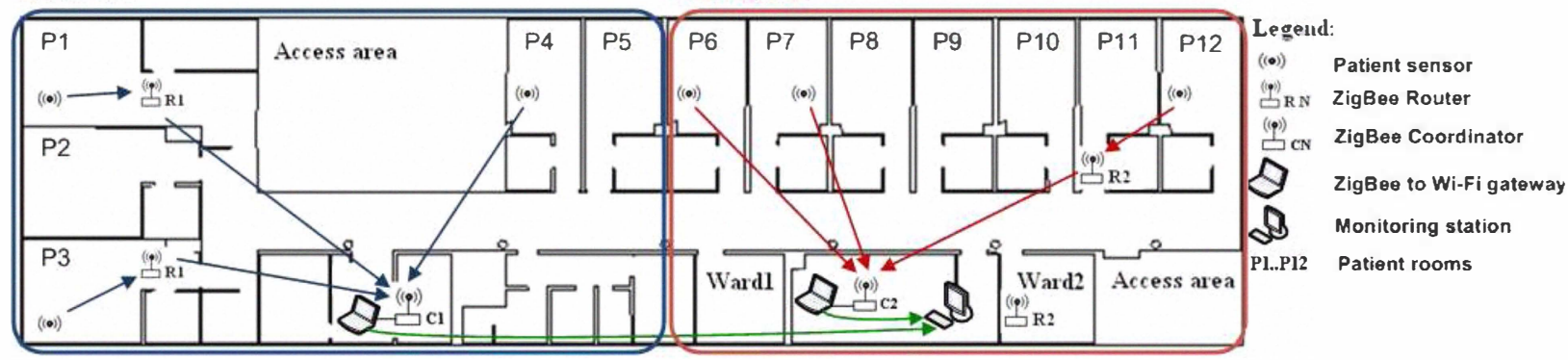

Figure 3. HM4All topology.

\section{B. Wireless Sensors}

HM4All specifications included ECG waveform, heart rate, $\mathrm{SpO} 2$, and skin temperature monitoring. ECG and temperature sensors initial versions are already prototyped. A $\mathrm{SpO} 2$ sensor is being designed. All sensors are based in the JN5139-M01 ZigBee module from Jennic [7]. A private profile (instead of a public profile defined by the ZigBee Alliance) was used.

Two versions of the ECG sensor were designed; both are adequate for monitoring purposes, and are based on the INA118 instrumentation amplifier from Texas Instruments. Analog filters are used to remove components outside of the $0.5 \mathrm{~Hz}-40 \mathrm{~Hz}$ band; an additional analog notch filter was used to reduce the $50 \mathrm{~Hz}$ power line noise. The 12-bit ADC internal to the communication module was used to sample the signal at $200 \mathrm{~Hz}$. The second version, which is being prototyped, uses a little bigger lithium battery to extend the sensor's autonomy, allowing a lifetime greater than seventy two hours when ECG signals are continuously transmitted.

The heart shape on the prototyped case shown in Figure 4 (a) indicates the correct orientation when attached to the patient's body to present the lead I ECG signal. As shown in Figure 4 (b), there are no cables between the sensor and the electrodes, what reduces the noise and makes it less intrusive, as pointed out by the 72-year-old female patient shown in Figure 4 (c), who was simultaneously being monitored using our ECG sensor and a commercial bedside monitoring equipment. Figure 5 (a) shows the ECG waveform from a young female subject at rest measured at the output of the sensor using an oscilloscope. Figure 5 (b) shows the signal presented by the application running in the monitoring station.

The skin temperature sensor was designed to use $2250 \Omega$ NTC thermistors probes. The sensor precision is highly dependent of the probe used. If medical grade probes are employed, a precision equal to $\pm 0.1{ }^{\circ} \mathrm{C}$ in the $34{ }^{\circ} \mathrm{C}$ to $43{ }^{\circ} \mathrm{C}$ temperature range can be achieved.

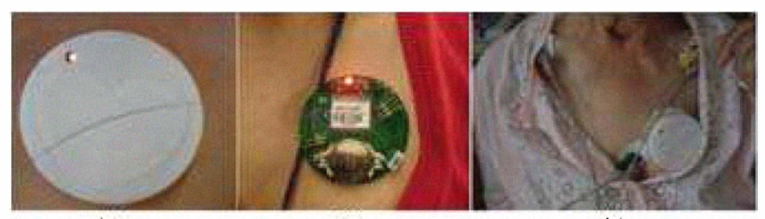

(a)

(b)

(c)

Figure 4. ECG sensor: (a) case; (b) board; (c) being used by a patient.

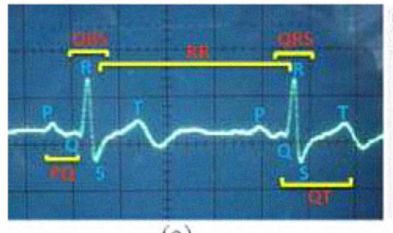

(a)

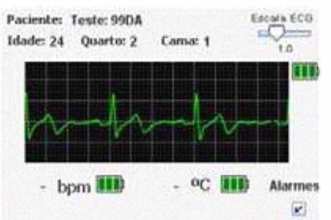

(b)
Figure 5. ECG signal: (a) measured in the lab and (b) displayed in the nurses' station. Scale: $0.2 \mathrm{~s} / \mathrm{div} ; 0.5 \mathrm{~V} / \mathrm{div}$.

A textile band is used to hold the temperature sensor case around the patient's arm, close to the armpit, where the temperature probe can be placed. Every two minutes a new skin temperature reading is sent. As with the ECG sensor, every ten minutes it also sends the measured battery voltage. Based on this value, the application running in the nurses' station can monitor the battery level.

\section{Software Applications}

The ZigBee-to-Wi-Fi Gateway, which was developed in $\mathrm{CH}$, contains two basic modules. The first one is responsible for establishing a serial connection, verifying the integrity and correctness of the data and processing them while the other one is responsible for establishing HTTP connections to send the processed data to the database as well as exhibiting relevant sensor related information to the system administrator.

The Data Visualization Application that runs in the Data Server (see Figure 2) is a Web-based application developed in Java. It consists of two servlets: the Data Reception Service (DRS) and the Data Dispatch Service (DDS). The DRS receives and validates processed data received from the ZigBee-to-Wi-Fi Gateway. It also stores data on the database. The DDS is responsible for serving data to authenticated clients. Both applications are supported by the Apache Tomcat Web server and the MySQL database. Additionally, a Web site was developed to allow patient data visualization and to provide several additional functionalities to authenticated users, such as:

- Patient registration, sensor insertion, and association between patients and sensors;

- Individual alarm configuration; and

- Historical data presentation in graphical format. 


\section{RESULTS}

As already stated, the prototype system was designed to monitor up to six patients simultaneously. The packet delivery ratio observed in tests realized using six ECG and six temperature sensors in the hospital floor is close to $100 \%$. Although results are encouraging, and confirm simulation data [8], more tests are necessary to validate system performance because, as the hospital is not yet fully operational, some sources of electromagnetic interference are not present.

\section{RELATED WORK}

Our work shares similarities with the SMART system [9] and a monitoring system developed at University of Texas (UT) [10]. SMART can monitor SpO2, ECG, and location of multiple patients. A commercial $\mathrm{SpO} 2$ sensor was used while the ECG sensor was developed as a Cricket mote daughter board. Vital signs and location data are sent to a PDA using a serial wired connection. All information gathered is transmitted using Wi-Fi to a central unit with signal processing and data analysis capability. Alarms can be sent to caregivers' PDAs. Similarly to our approach, raw ECG data is also transmitted. In our approach, however, we do not use wired BANs, but fully wireless sensors connected to an infrastructure distributed throughout the hospital. The UT system was developed to transmit vital signals through a ZigBee multi-hop network. To verify the system functionalities, a commercial blood pressure and heart-rate monitor was interfaced to a wireless sensor node, which commands the monitor to initiate a reading, collects the data and forwards them to the base station. Patient data are stored and displayed on the base station. The network topology chosen by us limits the number of hops of the ZigBee network because, as shown in [8], the depth of the network can seriously restricts the amount of data that can be relayed by the network. This concern is not shared by UT researchers as in their system short messages are infrequent.

In [11], the developed system aims at transmitting vital signals from in-patients in a very restricted area: an intensive care unit. This scenario differs from ours because, in our case, patient mobility obliges the system to provide means of efficiently collecting the signals throughout the hospital targeted areas without losing information.

For rescue and care delivery in remote and rural areas, researchers have proposed the use of technologies primarily designed to carry voice or video, such as GSM/GPRS, 3G and satellite technologies [12]. Many researchers who have considered disaster scenarios have proposed the use of BANs to primarily collect data from wearable sensors and relay them to another network. CodeBlue [13], BlueBio [14] and AID-N [15] are among the most interesting systems proposed.

\section{CONCLUSIONS AND FUTURE WORK}

We have presented HM4All, a prototype monitoring system being deployed in a hospital in Portugal. Presently, the system is able to monitor ECG, heart rate and skin temperature. A $\mathrm{SpO} 2$ sensor is under development. The system employs an easily scalable topology based on spatially distributed ZigBee networks that allow sensors to communicate directly with network devices and eliminate the compulsory personal devices carried by patients in systems based on BANs.
The developed Web-based applications allow information to be displayed in any computer with access to the Internet, and a PDA-based application is under development. Simulations and measurements support the view that the system is able to provide adequate system performance under the expected load.

Our future work includes tests to be carried out when the hospital is fully operational, deeply investigating ways of overcoming electromagnetic sources of interference in the hospital environment and comparing the performance of distributed networks with a topology based on BANs.

\section{ACKNOWLEDGMENTS}

Work supported by Fundação para a Ciência e a Tecnologia, under grant SFRH/BD/39408/2007 and through the $3^{\circ}$ Quadro Comunitário de Apoio and the POCTI and FEDER programmes, and by Casa de Saúde de Guimarães.

\section{REFERENCES}

[1] HealthGrades, "HealthGrades Hospital Quality in America Study," 2009

[2] H. Fernandez-Lopez, "Interview with Warren Sandberg, MD, PhD Monitoring Systems: Issues and Improvements," Boston, MA 2009.

[3] K. Holger and A. Willig, Protocols and Architectures for Wireless Sensor Networks NJ: Willey, 2005.

[4] IEEE, "IEEE Std 802.15.4-2003-IEEE Standard for Information Technology-Local and metropolitan area networks-Specific requirements--Part 15.4," IEEE, Ed., 2003

[5] ZigBee.Alliance, "ZigBee Alliance Document 053474r17, ZigBee Specification, v. 1.0 r17," Z. Alliance, Ed., 2007.

[6] B. Haile, "Wireless Sensors and Control Networks: Enabling New Opportunities with ZigBee," in ZigBee Alliance Tutorial, Dec 2006.

[7] Jennic, "Product Brief - JN5139-xxx-Myy IEEE802.15.4/ZigBee Module Family," 2008

[8] H. Fernandez-Lopez, P. Macedo, J. A. Afonso, J. H. Correia, and R. Simões, "Evaluation of the Impact of the Topology and Hidden Nodes in the Performance of a ZigBee Network," in S-Cube 2009. vol. LNICST 24, Italy: ICST, 2009, pp. 256-271.

[9] C. Dorothy, S. Eugene, W. Jason, G. John, B. Jacob, S. Thomas, A. G. Robert, and O.-M. Lucila, "Physiological signal monitoring in the waiting areas of an emergency room," in Proc. ICST $3^{\text {rd }}$ International Conf. on Body Area Networks Tempe, AZ: ICST, 2008

[10] A. Hande, T. Polk, W. Walker, and D. Bhatia, "Self-Powered Wireless Sensor Networks for Remote Patient Monitoring in Hospitals," Sensors vol. 6, pp. 1102-1117, 22 September 2006.

[11] N. O'Donoughue, S. Kulkarni, and D. Marzella, "Design and Implementation of a Framework for Monitoring Patients in Hospitals Using Wireless Sensors in Ad Hoc Configuration," in 28th IEEE EMBS Annual Intl Conf. New York City, USA: IEEE, 2006, pp. 6449-6452.

[12] E. Kyriacou, M. S. Pattichis, A. Panayides, and A. Pitsillides, "m-Health e-Emergency Systems: Current Status and Future Directions," Antennas and Propagation Magazine, IEEE, vol. 49, pp. 216-231, 2007.

[13] K. Lorincz, D. J. Malan, T. R. F. Fulford-Jones, A. Nawoj, A. Clavel, V. Shnayder, G. Mainland, M. Welsh, and S. Moulton, "Sensor networks for emergency response: challenges and opportunities," Pervasive Computing, IEEE, vol. 3, pp. 16-23, 2004

[14] G. Kramp, M. Kristensen, and J. F. Pedersen, "Physical and digital design of the BlueBio biomonitoring system prototype, to be used in emergency medical response," in Pervasive Health Conference and Workshops, 2006, 2006, pp. 1-11.

[15] G. Tia, C. Pesto, L. Selavo, C. Yin, K. Jeong Gil, L. Jong Hyun, A. Terzis, A. Watt, J. Jeng, C. Bor-rong, K. Lorincz, and M. Welsh, "Wireless Medical Sensor Networks in Emergency Response: Implementation and Pilot Results," in Technologies for Homeland Security, 2008 IEEE Conference on, 2008, pp. 187-192. 